\title{
The Effect of Pain Reduction during Headframe Fixation for Stereotactic Radiosurgery by the Preceded Local Anesthesia under the Needle Cap Guidance
}

\author{
Dong Hwan Kim¹, Won Ho Cho ${ }^{1}$, Beong lk Hur ${ }^{1}$, Seung Heon Cha', Sang Weon $\mathrm{Lee}^{2}$, Chang Hwa Choi ${ }^{1}$ \\ 'Department of Neurosurgery \& Medical Research Institute, Pusan National University Hospital, Pusan National University School of Medicine, \\ Busan, Korea \\ ${ }^{2}$ Department of Neurosurgery, Pusan National University Yangsan Hospital, Pusan National University School of Medicine, Yangsan, Korea
}

Received June 4, 2019

Revised June 27, 2019

Accepted June 28, 2019

\section{Correspondence}

Won Ho Cho

Department of Neurosurgery,

Pusan National University Hospital,

179 Gudeok-ro, Seo-gu,

Busan 49241, Korea

Tel: $+82-51-240-7257$

Fax: +82-51-244-0282

E-mail: mdcwh@naver.com
Background Gamma knife radiosurgery (GKRS) has become a major alternative in the neurosurgical field. However, many patients complained of considerable discomfort during the fixation of rigid headframe. This study investigated whether our modified procedure could reduce fixation-related pain.

Methods Sixty-six patients who underwent GKRS were enrolled in this study. Thirty-one patients (Group A) underwent the conventional subcutaneous infiltration technique, and 35 patients (Group B) did the modified procedure. In group A, the headframe was held in position by an assistant, and local anesthetics were injected subcutaneously using a 23-gauge spinal needle at pinning sites. Subsequently, pins were applied according to measurements based on spinal needle depth. In group $\mathrm{B}$, with the frame held in position by an assistant, pin sites were marked with a surgical pen under the guidance of needle cap placed on the pin holes. The head frame was then removed, and local anesthetics were injected subcutaneously and periosteally at each marked pin site using a 26-gauge needle. The headframe was then repositioned 5 minutes after local infiltration, and pins were applied according to measurements based on spinal needle depth. To evaluate pain severity during procedures, visual analogue scale (VAS) scores were recorded during local infiltration and frame placement with pins. The pain scores of the two groups were analyzed statistically.

Results Group B had a significantly lower VAS score during frame placement than group A (7.26 vs. $3.61 ; p<0.001)$, and mean VAS score at local infiltration was also significantly lower in group B (4.74 vs. $3.74 ; p=0.008)$.

Conclusion Patients in group B experienced significantly less pain than those in group A during pin placement. Pre-fixation time advanced local anesthesia might reduce pain during stereotactic procedures, and the use of a 26-gauge needle appeared in less pain during local infiltration.

Key Words Stereotactic radiosurgery; Gamma knife radiosurgery; Pain.

\section{INTRODUCTION}

Stereotactic radiosurgery is being rapidly adopted for the treatment of various neurosurgical diseases including brain tumors, vascular malformations, trigeminal neuralgia, and even Parkinson's disease due to its safety and effectiveness [1-5]. The

This is an Open Access article distributed under the terms of the Creative Commons Attribution Non-Commercial License (https://creativecommons.org/licenses/by-nc/4.0) which permits unrestricted non-commercial use, distribution, and reproduction in any medium, provided the original work is properly cited.

Copyright ( $\odot 2019$ The Korean Brain Tumor Society, The Korean Society for NeuroOncology, and The Korean Society for Pediatric Neuro-Oncology accurate delivery of ionizing radiation to a specific focus as previously determined by MRI or CT is important for the effective and accurate treatment of intracranial lesions. Therefore, accurate headframe placement is a critically important aspect of gamma knife radiosurgery (GKRS). Headframe is rigidly attached to patients' skull to establish a stereotactic coordination system and provide a means for precisely positioning the patient in stereotactic space. For example, the Leksell headframe (Elekta AB, Stockholm, Sweden) is fixed to the outer table of skull with two anterior and two posterior screws [6].

Unfortunately, most patients experience considerable dis- 
comfort during frame fixation, and this discomfort has been overlooked, because surgeons naturally have focused on successfully conducting the procedure, sustaining stable vital signs, and avoiding problems. Nevertheless, reducing pain during frame fixation would reduce patient discomfort and tend to stabilize vital signs.

Traditionally, local anesthetic solution is injected subcutaneously at anticipated pinning sites using a 23-gauge spinal needle, while the stereotactic frame is held in position by an assistant. However, most patients complain of considerable pain despite of local anesthesia.

This study was designed to quantify and compare levels of pain experienced by patients when stereotactic headframes are placed using the conventional method or modified method designed to reduce pain during headframe fixation.

\section{MATERIALS AND METHODS}

We retrospectively studied 66 consecutive patients that underwent GKRS to treat intracranial brain lesions at either of two institutes between April 2015 and December 2015. This study was reviewed and approved by the Institutional Review Board of the Pusan National University Hospital (IRB No: H-1903-009-076). Patients that underwent GKRS due to trigeminal neuralgia and those receiving opioid medication to control pain were excluded. Demographic and baseline variables were collected including age at time of GKRS, gender, diagnosis, degree of pain [as assessed using visual analogue scale (VAS)] during intramuscular (IM) injections based on patients' past experiences. We also excluded patients with a VAS score of 0 during IM injection. For analytic purposes, diagnoses were categorized as brain tumors or vascular malformations. Brain tumors consisted of metastatic brain tumors, meningiomas, schwannomas, lymphomas, and gangliogliomas, and vascular malformations consisted of arteriovenous and cavernous malformations.

The 66 study subjects were divided into two groups according to the method of stereotactic frame fixation used. Group A consisted of 31 patients that received the conventional subcutaneous infiltration technique, and group B consisted of 35 patients that received the modified procedure.

The patients in group A received usual subcutaneous infiltration. Briefly, with the headframe held in position by an assistant, local anesthetic solution (2\% lidocaine hydrochloride, 2-3 $\mathrm{mL}$ per pinning site) was injected subcutaneously at 4 pinning sites using a 23 -gauge spinal needle. The assistant was hard to hold for a long time with the headframe, the 4 pins were applied immediately after local anesthesia according to measurement based on spinal needle depth. No patient received intravenous fentanyl or midazolam prior to headframe placement.

In group B, we performed local anesthesia using modified procedure during stereotactic frame fixation. Briefly, with the headframe held in a standard position by an assistant, each of anticipated pin sites was marked with surgical pen using a needle cap located in bar attached a headframe (Fig. 1). After marking pin sites, the stereotactic frame was removed, and local anesthetic solution (2\% lidocaine hydrochloride, 2-3 $\mathrm{mL}$ per pinning site) was injected periosteally and subcutaneously at each marked pin site using a 26-gauge sharp-beveled needle (Fig. 2). The stereotactic frame was repositioned by an assistant under the same guidance of needle cap using pin site marks 5 minutes after local infiltration and gentle massage at

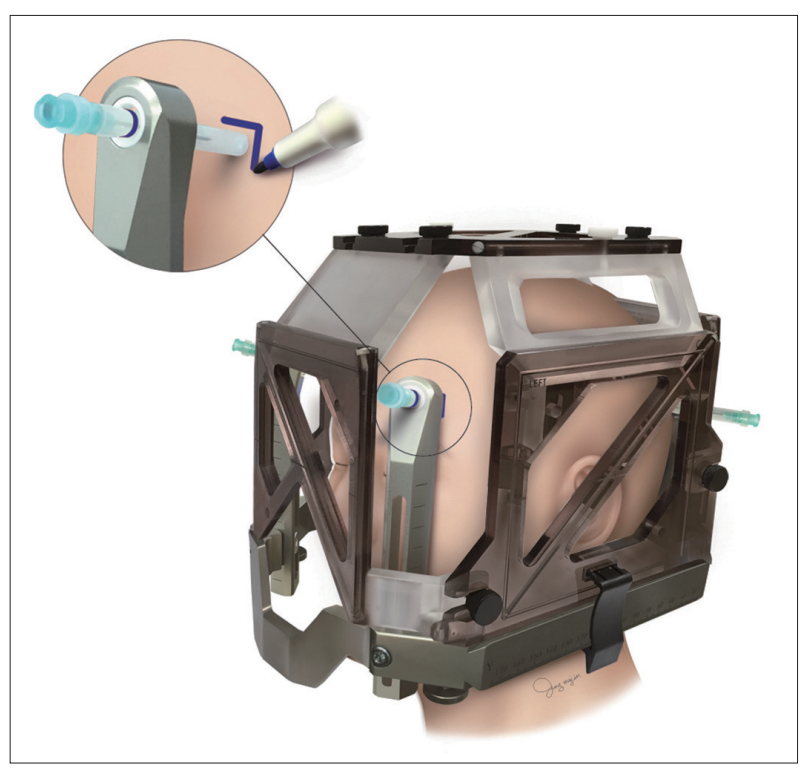

Fig. 1. Pin sites were marked with surgical pen under the guidance of needle cap positioned through the pin hole in the frame bar.

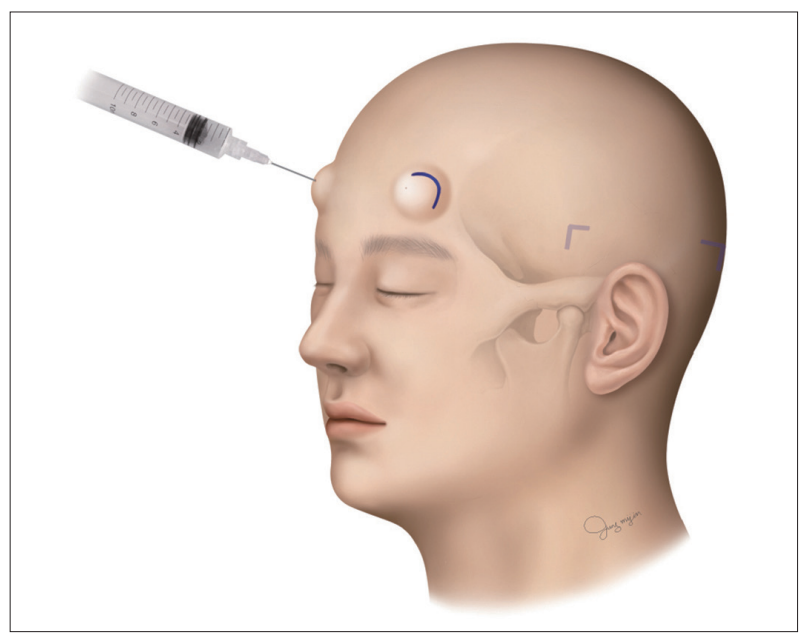

Fig. 2. Local anesthetic solution was injected periosteally and subcutaneously at each marked pin site using a 26 gauge sharpbeveled needle. 


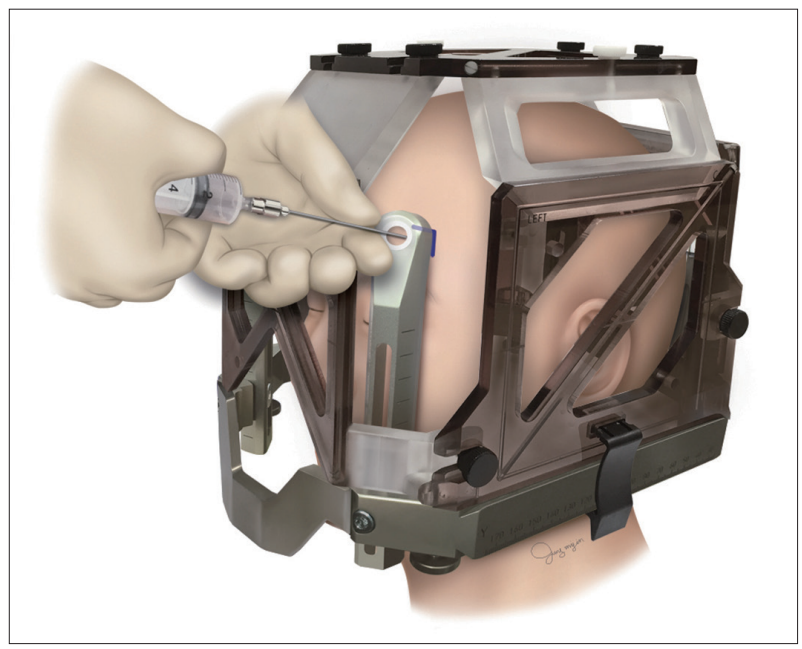

Fig. 3. Pin length was measured by spinal needle depth, before applying to the marking sites of patient head.

areas of intended local infiltration. Pins were applied simultaneously to marked sites according to measurement based on spinal needle depth (Fig. 3). No patients received intravenous fentanyl or midazolam prior to frame placement.

To evaluate the severity of pain during the procedures, patients' VAS (scale: $0-10$ ) were assessed twice, that is, during local infiltration and pin insertion. In addition, to minimize group bias, we obtained VAS during IM injection based on patients' recollections. VAS of two groups was compared using the independent t-test. Statistical analysis was performed using SPSS version 18.0 for Windows (SPSS Inc., Chicago, IL, USA). $p$ values of less than 0.05 were considered statistically significant.

\section{RESULTS}

Sixty-six patients were enrolled in this study. There were 16 male and 15 female patients in Group A $(n=31)$ and 19 male and 16 female patients in Group B $(n=35)$. For the 66 study subjects, mean average age was 56.4 years old and there were slightly more men $(n=35)$ than women $(n=31)$. The most common pathology treated was metastatic brain tumors $(n=39)$. Age, gender, and diagnosis were non-significantly different in the two groups. Demographic characteristics are shown in Table 1.

The results obtained that mean VAS during headframe placement were significantly lower in group B than in group A (3.61 vs. 7.26 ; $p<0.001)$. Additionally, mean VAS score was also significantly lower during local injection in Group B ( 3.74 vs. $4.74 ; p=0.008$ ). However, mean group VAS scores at IM injection were non-significantly different $(p=0.24)$. These findings are summarized in Table 2.
Table 1. Demographic data of the study subjects

\begin{tabular}{lcccc}
\hline & $\begin{array}{c}\text { Total } \\
(\mathrm{n}=66)\end{array}$ & $\begin{array}{c}\text { Group A } \\
(\mathrm{n}=31)\end{array}$ & $\begin{array}{c}\text { Group B } \\
(\mathrm{n}=35)\end{array}$ & $p$-value \\
\hline $\begin{array}{l}\text { Age }(\mathrm{yr}), \\
\text { mean } \pm \text { SD }\end{array}$ & $56.4 \pm 13.9$ & $56 \pm 13.3$ & $56.8 \pm 14.6$ & 0.590 \\
$\begin{array}{l}\text { Gender } \\
\text { Male }\end{array}$ & 35 & 16 & 19 & 0.704 \\
Female & 31 & 15 & 16 & \\
Diagnosis & & & & 0.897 \\
Brain group & & & & \\
$\quad$ Metastasis & 39 & 14 & 25 & \\
$\quad$ Meningioma & 9 & 8 & 1 & \\
$\quad$ Schwannoma & 5 & 3 & 2 & \\
Lymphoma & 1 & 0 & 1 & \\
Ganglioglioma & 1 & 0 & 1 & \\
Vascular group & & & & \\
AVM & 5 & 3 & 2 & \\
CM & 6 & 3 & 3 & \\
\hline AVM, arteriovens & & & & \\
\hline
\end{tabular}

$\mathrm{AVM}$, arteriovenous malformation; $\mathrm{CM}$, cavernous malformation

Table 2. Comparisons of group mean visual analogue scale scores

\begin{tabular}{lccc}
\hline & Group A & Group B & $p$-value \\
\hline IM injection & $2.67 \pm 1.24$ & $2.31 \pm 1.23$ & 0.240 \\
During local injection & $4.74 \pm 1.46$ & $3.74 \pm 1.49$ & 0.008 \\
During frame placement & $7.26 \pm 1.95$ & $3.61 \pm 1.95$ & $<0.001$ \\
\hline
\end{tabular}

Results are presented as means \pm SDs.

\section{DISCUSSION}

Nowadays, GKRS is a major therapeutic modality in the neurosurgical field, and an accepted safe, effective procedure. However, many patients experience considerable pain and anxiety during stereotactic frame application, and some complain bitterly. Nevertheless, little attention has been paid to the pain that occurs when a frame is fixed and few studies have addressed the topic.

Liang et al. [7] reported on the effectiveness of topical anesthetic EMLA for postoperative wound pain after GKRS, but Duenas et al. [8] reported that topical EMLA did not significantly reduce pain when used preoperatively for frame fixation. Watson et al. [9] compared the effects of supraorbital and great occipital nerve block and subcutaneous infiltration on pain during stereotactic frame placement, and concluded supraorbital and greater occipital nerve block offered alternatives to subcutaneous infiltration for stereotactic frame placement, although no significant difference was detected between subcutaneous infiltration and nerve block. Wang et al. [6] investigated patient pain, when angled anterior posts were used to avoid temporalis muscle penetration 
during stereotactic headframe placement, but found no significant difference between conventional anterior posts and angled anterior posts.

The present study was used to objectively assess pain associated with stereotactic frame fixation and compare the pain associated with conventional frame fixation with that experienced using modified frame fixation method. In Group A, while maintaining the stereotactic frame, subcutaneous infiltration was performed at pinning sites using a 23-gauge spinal needle, and then frame fixation was performed promptly. This is because the assistant is hard to keep holding the headframe without changing its location. In Group B, after marking pinning sites with a surgical marking pen under frame guidance, the frame was removed and a local anesthetic solution was injected into the periosteal and subcutaneous layers at marked sites using a 26-gauge sharp-beveled needle. The headframe was repositioned 5 minutes later using the pen marks of patient's head and the 4 pins were applied. Mean VAS at local injection and frame fixation was found to be significantly lower in Group B. VAS was also obtained for IM injections based on patient recollections and no significant intergroup difference was found $(p=0.24)$.

We suggest several hypotheses for the lower mean VAS in the group B. First, the onset time of local anesthesia will be important. Lidocaine is known to have an onset about 2 minutes after injection. So the timing of frame fixation and the technique of local anesthesia in group A might have been detrimental to its success for pain reduction during pin placement, relative to group B. Local anesthesia was performed without frame placement into subcutaneous and periosteal spaces in group B. In addition, when our technique is used, it would also be possible to inject several times around marked pinning sites using the pivot technique, and headframe fixation could also be performed after the local anesthetic has taken effect. On the other hand, subcutaneous infiltration was performed while the assistant held the headframe in group A, and pin placement was performed before the anesthetic had taken effect. Furthermore, local anesthetic was injected at a single point during the subcutaneous infiltration technique, which raised a tight bleb, and this is probably painful because scalp tissues are dense and the local increase in tissue pressure is likely to be considerable [9].

Second, the course of the supraorbital nerve and depth of local anesthesia might have affected pain severity. The supraorbital nerve usually exits via the supraorbital foramen in the medial orbital rim and then branches into medial and lateral branches. The medial branch crosses and runs superficially over frontal muscle to supply the forehead skin and anterior scalp as far as the vertex, whereas the lateral branch lateralizes, traveling on periosteum $1 \mathrm{~cm}$ medial and parallel to the tempo- ral crest and enters the galea in the cranial portion of the forehead, providing sensitivity to the parietal region [10-12]. When an anesthetic solution is injected, it is considered to have been injected into the subcutaneous space, but in most cases, an anesthetic solution is delivered after the inserted spinal needle touches bone, and thus, the anesthetic solution is injected into the periosteal space rather than the subcutaneous space, which causes a bleb-like cyst (Fig. 4). This may be due to difficulty of controlling the penetration depth with a relatively long spinal needle. Therefore, injecting anesthetic agents into the periosteal space does not result in sufficient anesthetization of the medial branch of the supraorbital nerve, and thus, does not adequately relieve pain during pinning. Thus, in our opinion injecting anesthetic solution into the subcutaneous and periosteal spaces

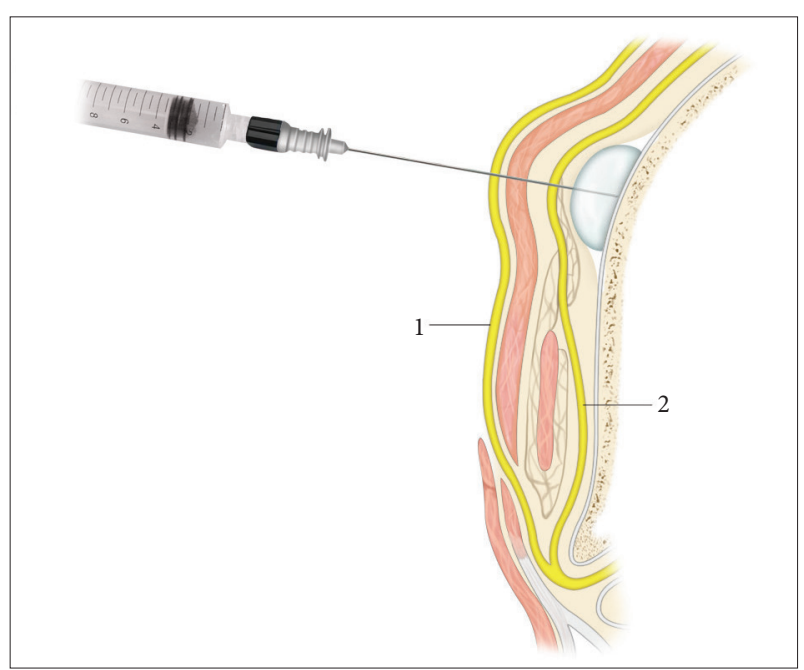

Fig. 4. Anesthetic solution was injected into the periosteal space and formed a bleb-like cyst. 1 , medial branch of the supraorbital nerve; 2 , lateral branch of the supraorbital nerve.

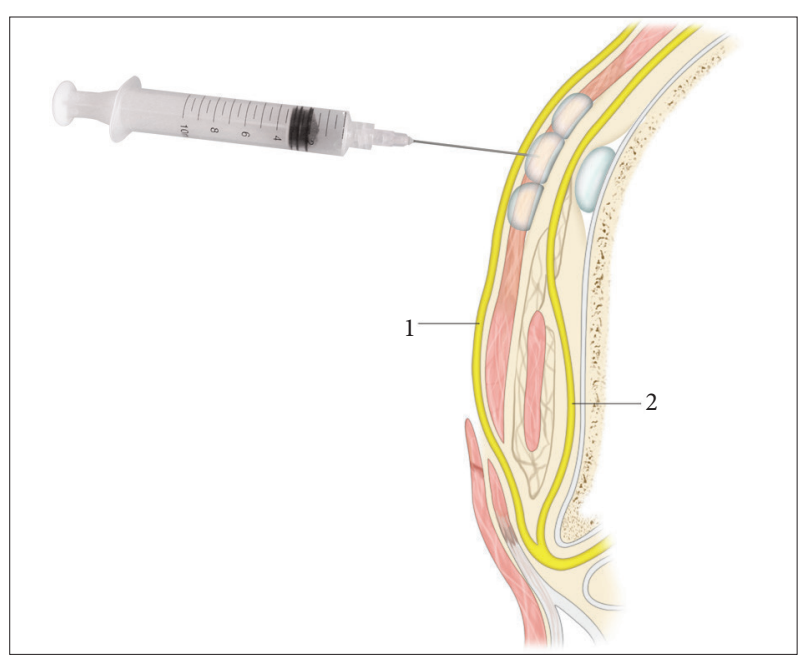

Fig. 5. Anesthetic solution was injected into the periosteal and subcutaneous spaces to anesthetize medial and lateral branches of the supraorbital nerve. 1 , medial branch of the supraorbital nerve; 2 , lateral branch of the supraorbital nerve. 
helps reduce pain (Fig. 5). Viewed in the course of greater and lesser occipital nerve, difference of pain degree in occiput between two groups is similar with that in forehead.

Third, a 26-gauge sharp-beveled needle was used in group $\mathrm{B}$, which would have reduced pain at time of skin penetration as compared with the 23-gauge needle used in group A.

In conclusion, proper stereotactic frame fixation is of considerable importance for successful GKRS, but pain reduction when fixing the frame also requires consideration. In the present study, we found that our modified method could be helpful for reducing pain and discomfort during the stereotactic procedure.

\section{Conflicts of Interest}

The authors have no potential conflicts of interest.

\section{Acknowledgments}

This work was supported by a 2-year Research Grant of Pusan National University.

\section{REFERENCES}

1. Guridi J, Obeso JA, Rodriguez-Oroz MC, Lozano AA, Manrique M. L-dopa-induced dyskinesia and stereotactic surgery for Parkinson's disease. Neurosurgery 2008;62:311-23; discussion 323-5.

2. Kondziolka D, Lunsford LD, Flickinger JC. The application of stereotactic radiosurgery to disorders of the brain. Neurosurgery 2008;62
Suppl 2:707-19; discussion 719-20.

3. Kondziolka D, Martin JJ, Flickinger JC, et al. Long-term survivors after gamma knife radiosurgery for brain metastases. Cancer 2005; 104:2784-91.

4. Kondziolka D, Perez B, Flickinger JC, Habeck M, Lunsford LD. Gamma knife radiosurgery for trigeminal neuralgia: results and expectations. Arch Neurol 1998;55:1524-9.

5. Nicolato A, Foroni R, Pellegrino M, et al. Gamma knife radiosurgery in meningiomas of the posterior fossa. Experience with 62 treated lesions. Minim Invasive Neurosurg 2001;44:211-7.

6. Wang DD, Lau D, Rolston JD, Englot DJ, Sneed PK, McDermott MW Pain experience using conventional versus angled anterior posts during stereotactic head frame placement for radiosurgery. J Clin Neurosci 2014;21:1538-42.

7. Liang CL, Lu K, Liliang PC, Chung MC, Chi SC, Chen HJ. Topical anesthetic EMLA for postoperative wound pain in stereotactic gamma knife radiosurgery: a perspective, randomized, placebo-controlled study. Minim Invasive Neurosurg 2011;54:75-8.

8. Duenas SM, Pun J, Radwan HA, Akerman M, Schulder M. A randomized trial on the efficacy of topical anesthesia for pain reduction during frame placement for gamma knife radiosurgery. Stereotact Funct Neurosurg 2016;94:259-64.

9. Watson R, Leslie K. Nerve blocks versus subcutaneous infiltration for stereotactic frame placement. Anesth Analg 2001;92:424-7.

10. Konofaos P, Soto-Miranda MA, Ver Halen J, Fleming JC. Supratrochlear and supraorbital nerves: an anatomical study and applications in the head and neck area. Ophthalmic Plast Reconstr Surg 2013;29:403-8.

11. Knize DM. A study of the supraorbital nerve. Plast Reconstr Surg 1995;96:564-9.

12. Malet T, Braun M, Fyad JP, George JL. Anatomic study of the distal supraorbital nerve. Surg Radiol Anat 1997;19:377-84. 